\title{
Preparation, Structural, Electrical, and Ferroelectric Properties of Lead Niobate-Lead Zirconate-Lead Titanate Ternary System
}

\author{
Rashmi Gupta, Seema Verma, Vishal Singh, and K. K. Bamzai \\ Crystal Growth \& Materials Research Laboratory, Department of Physics and Electronics, University of Jammu, \\ Jammu 180006, India \\ Correspondence should be addressed to K. K. Bamzai; kkbamz@yahoo.com
}

Received 8 September 2014; Revised 2 January 2015; Accepted 9 January 2015

Academic Editor: Shaomin Liu

Copyright (C) 2015 Rashmi Gupta et al. This is an open access article distributed under the Creative Commons Attribution License, which permits unrestricted use, distribution, and reproduction in any medium, provided the original work is properly cited.

A ternary system of lead niobate-lead zirconate-lead titanate with composition $x \mathrm{PN}-y \mathrm{PZ}-(x-y) \mathrm{PT}$ where $x=0.5$ and $y=0.15$, 0.25 , and 0.35 known as PNZT has been prepared by conventional mixed oxide route at a temperature of $1100^{\circ} \mathrm{C}$. The formation of the perovskite phase was established by X-ray diffraction analysis. The surface morphology studied by scanning electron microscopy shows the formation of fairly dense grains and elemental composition was confirmed by energy dispersive X-ray analysis. Dielectric properties like dielectric constant and dielectric loss $\left(\varepsilon^{\prime}\right.$ and $\left.\tan \delta\right)$ indicate poly-dispersive nature of the material. The temperature dependent dielectric constant $\left(\varepsilon^{\prime}\right)$ curve indicates relaxor behaviour with two dielectric anomalies. The poly-dispersive nature of the material was analysed by Cole-Cole plots. The activation energy follows the Arrhenius law and is found to decrease with increasing frequency for each composition. The frequency dependence of ac conductivity follows the universal power law. The ac conductivity analysis suggests that hopping of charge carriers among the localized sites is responsible for electrical conduction. The ferroelectric studies reveal that these ternary systems are soft ferroelectric.

\section{Introduction}

Materials with high Curie temperature and piezoelectric properties are required for high temperature applications in industries. Barium titanate (BT) or lead zirconate titanate (PZT) is used for many commercial applications like medicine, industry, and research but due to their low Curie temperature, that is, $\sim-120^{\circ} \mathrm{C}$ for $\mathrm{BT}[1]$ and $\sim 390^{\circ} \mathrm{C}$ for PZT [2], they are not suitable for high temperature applications. Recently, a great deal of interest has been focused on relaxor ferroelectric materials due to their excellent piezoelectric as well as dielectric properties $[3,4]$. Lead metaniobate $\left(\mathrm{PbNb}_{2} \mathrm{O}_{6}\right.$ or $\left.\mathrm{PN}\right)$ reported by Goodman [5] is a member of tungsten bronze family and is often used in nondestructive testing and medical diagnostic imaging and for deep submergence hydrophones. However, problems such as high level of porosity and relatively low mechanical strength are often encountered in its use and since its discovery no much work has been done on it in spite of the high quality pioneering work $[6,7]$. Niobium based perovskite oxides have attracted much attention because they show combined electrical and mechanical properties for use in the electronic industries [8-10]. In spite of the reports of an attractive high Curie temperature $\left(517-570^{\circ} \mathrm{C}\right)[11,12]$, fewer studies [13-15] on PN have been carried out which can be attributed to the preparing of piezoelectric form (the metastable orthorhombic structure) in pure phase. On the other hand, one of the most widely studied ferroelectric materials due to its various potential technological applications is lead zirconate titanate, that is, $\mathrm{PbZr}_{1-x} \mathrm{Ti}_{x} \mathrm{O}_{3}$ (PZT) [16]. Aliovalent modifications to PZT which can be either with higher valence substitutions (donors) or with lower valence ions (acceptors) can alter its properties and have been studied by many authors [16, 17]. One of the most used additives is niobium which substitutes either $\mathrm{Zr}^{4+}$ or $\mathrm{Ti}^{4+}$, promotes the lead vacancies, and is considered as a donor dopant. Most of the studies have reported on the effect of niobium modification on the dielectric, piezoelectric, pyroelectric, and electrooptic properties of PZT in bulk as well as thin film form [18-25]. However, there are so many reports that due to lead evaporation during processing, both doped and undoped PZT, are seldom observed to be perfect [26-28]. In the present investigation, 


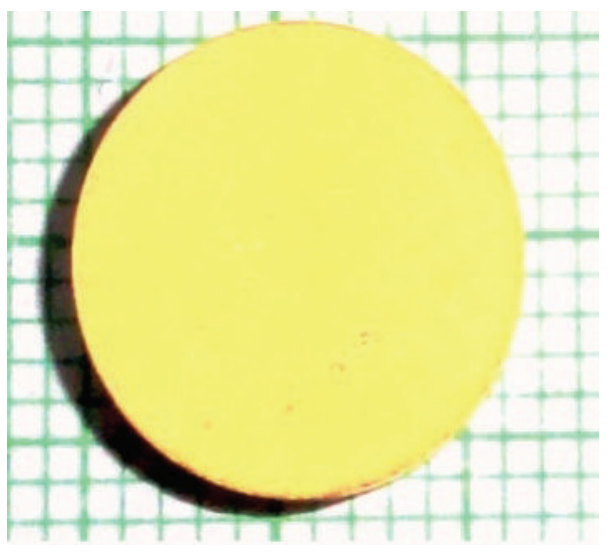

(a) $x=50, y=15 \%$

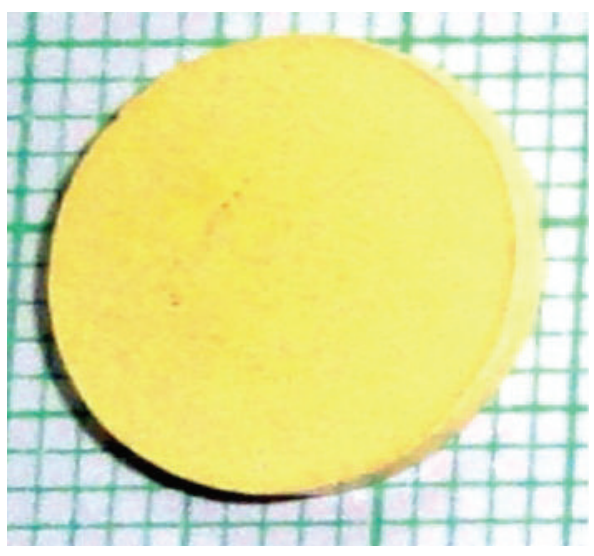

(b) $x=50, y=25 \%$

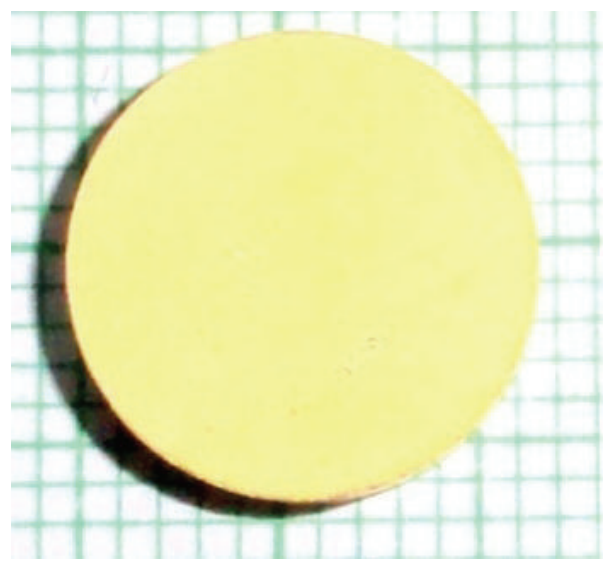

(c) $x=50, y=35 \%$

FIGURE 1: Photographs of the sintered pellets for $x \mathrm{PN}-y \mathrm{PZ}-(x-y) \mathrm{PT}$ (PNZT) $(x=50, y=15,25$, and 35\%).

we have investigated the preparation and properties of lead niobate-lead zirconate-lead titanate (PN-PZ-PT) ternary system. The effects on the electrical properties of the system have been investigated in detail by varying the PZ and PT concentration while keeping the PN concentration steady. To the best of author's knowledge, there is no such report on preparation and structural and electrical properties of such a ternary compound having general formula $(x)$ lead niobate $(\mathrm{PN})-(y)$ lead zirconate $(\mathrm{PZ})-(x-y)$ lead titanate (where $x=$ $0.5, y=0.15,0.25,0.35)$.

\section{Materials and Methods}

Polycrystalline ceramic of $x \mathrm{PN}-y \mathrm{PZ}-(x-y) \mathrm{PT}$ (where $x=$ $0.5, y=0.15,0.25$, and 0.35 ) popularly known as lead niobate zirconate titanate (PNZT) was prepared using the conventional mixed oxide route. Oxide powders of $\mathrm{PbO}$, $\mathrm{ZrO}_{2}, \mathrm{TiO}_{2}$, and $\mathrm{Nb}_{2} \mathrm{O}_{5}$ from S D Fine-Chem Limited (99\% pure) were used as raw materials. Stoichiometric amount as per the molecular formula was weighed and mixed together in a mortar and pestle first and then in a high energy ball mill, as well as homogenized with triple distilled water for $48 \mathrm{~h}$ using zirconia balls as the milling media. The mixture was then dried in an oven at $100^{\circ} \mathrm{C}$ for $2 \mathrm{~h}$. After drying, the reaction of the uncalcined powders taking place during heat treatment was investigated by thermogravimetric and differential thermal analysis (TGA-DTA, Shimadzu), using a heating rate of $10^{\circ} \mathrm{C} / \mathrm{min}$ in air from room temperature up to $1000^{\circ} \mathrm{C}$. Based on the TGA-DTA results, the mixture was calcined at a temperature of $900^{\circ} \mathrm{C}$ for a dwell time $2 \mathrm{~h}$ and heating/cooling rates ranging $4^{\circ} \mathrm{C} / \mathrm{min}$ in closed platinum crucible in order to ensure the proper chemical homogeneity of the constituent elements. The calcined powder was then grinded and mixed with $5 \mathrm{wt} \%$ of polyvinyl alcohol as binder and uniaxially pressed into pellets of $13 \mathrm{~mm}$ diameter by applying a pressure of $200 \mathrm{MPa}$ in a hydraulic press. These pellets were then sintered at $1100^{\circ} \mathrm{C}$ using a heating/cooling rate of $4^{\circ} \mathrm{C} / \mathrm{min}$ with dwell time of $4 \mathrm{~h}$ in a sealed platinum crucible. Figure 1 shows the photographs of the sintered ceramic pellets for all the three compositions considered in the present investigation. X-ray diffraction pattern was collected using a Rigaku X-ray diffractometer with $\mathrm{CuK} \alpha$ radiation $(\lambda=1.5405 \AA)$ and nickel filter in a wide $2 \theta$ range of $20-80^{\circ} \mathrm{C}$ using step scanning with a step size of $0.02^{\circ}$ and step time of $65.6 \mathrm{~s}$. The surface morphology and microstructures were examined using scanning electron microscope (SEM) of JEOL model number JSM-6390 LV and the presence of all the major elements was confirmed by energy dispersive 


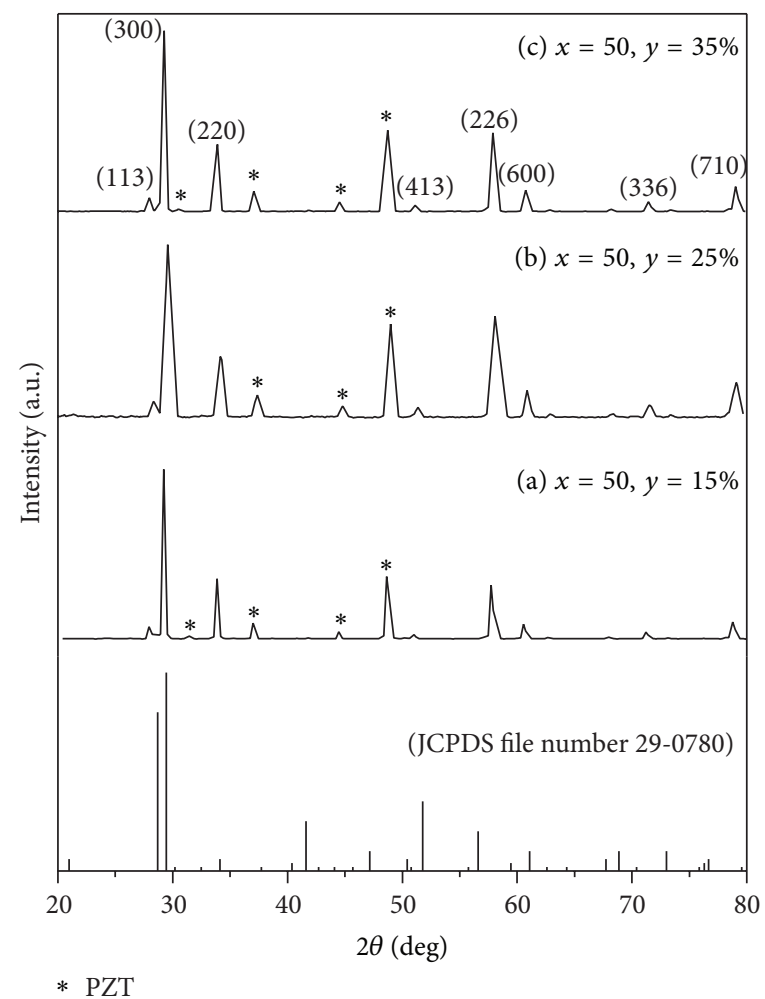

Figure 2: Room temperature X-ray diffraction pattern of $x \mathrm{PN}-y \mathrm{PZ}-(x-y) \mathrm{PT}$ ceramics sintered at $1100^{\circ} \mathrm{C}$ matched with JCPDS file number 29-0780 for the rhombohedral $\mathrm{PbNb}_{2} \mathrm{O}_{6} ;\left(^{*}\right)$ indicates the PZT phase.

$\mathrm{X}$-ray analysis (EDAX) of model number JEOL JED-2300. The dielectric measurements have been recorded with the help of automated impedance analyzer (4192A LF model) interfaced with USB-GPIB converter 82357 (Agilent) and further automated by using a computer for data recording, storage, and analysis. The polarization versus electric field ( $\mathrm{P}-$ E) hysteresis loop at room temperature was traced using an automatic PE loop tracer (Marine India Electrocom Ltd., New Delhi, India).

\section{Results and Discussion}

3.1. Structural Characterization. Figure 2 shows the X-ray diffraction (XRD) patterns of three different compositions of PNZT, namely, $x \mathrm{PN}-y \mathrm{PZ}-(x-y) \mathrm{PT}$ (where $x=0.5, y$ $=0.15,0.25$, and 0.35$)$ with a well-crystallized structure without the presence of pyrochlore or unwanted phases. The major diffraction peaks observed correspond to rhombohedral phase of $\mathrm{PbNb}_{2} \mathrm{O}_{6}$ (JCPDS 29 - 0780) with lattice parameters $a=10.501, \alpha=\beta=90^{\circ}, \gamma=120^{\circ}$, and space group $\mathrm{R} 3 \mathrm{~m}(160)[15,29]$. The peaks marked with star $\left(^{*}\right)$ in the figure correspond to PZT, thereby suggesting the presence of mixed phases in PNZT. The peaks were indexed according to the JCPDS card. The peaks with maximum intensity and maximum crystallite size were observed for the composition having 35\% zirconium which shows the improved crystallinity of the material. The backscattered scanning electron micrograph (SEM) images are shown in Figures 3(a)-3(c).
The micrographs clearly show the formation of fairly dense grains and the average grain size calculated using linear intercept method was found to be $1.40,1.46$, and $1.18 \mu \mathrm{m}$ for 15,25 , and $35 \%$ of zirconium content, respectively. The elemental composition was analyzed by energy dispersive Xray analysis (EDAX) (Figures 4(a)-4(c)) which confirms the presence of almost all the constituent elements, that is, $\mathrm{Pb}, \mathrm{Nb}$, $\mathrm{Ti}, \mathrm{Zr}$, and $\mathrm{O}$ in the composition. Table 1 shows the grain size, crystalline size calculated by Debye Scherrer formula [30] as well as Williamson-Hall plot [31], and strain and crystallinity index for all the three compositions. Crystallinity is evaluated from the crystallinity index equation [32]:

$$
I_{\text {cry }}=\frac{D_{p}(\mathrm{SEM}, \mathrm{TEM})}{D_{\text {cry }}(\mathrm{XRD})}, \quad I_{\text {cry }}(\geq 1.00),
$$

where " $I_{\text {cry }}$ " is the crystallinity index; " $D_{p}$ " is the particle size (obtained from either TEM or SEM morphological analysis); " $D_{\text {cry }}$ " is the particle size (calculated from the Scherrer equation). If " $I_{\text {cry }}$ " value is close to 1 , then it is assumed that the crystallite size represents monocrystalline whereas a polycrystalline has a much larger crystallinity index.

3.2. Thermal Analysis. The result of thermogravimetric analysis (TGA) and differential thermal analysis (DTA) of a milled powder having the composition $0.50 \mathrm{PN}-0.25 \mathrm{PZ}-$ $0.25 \mathrm{PT}$ is shown in Figure 5. Three endothermic peaks followed by small weight loss were observed in the DTA curve in the temperature range $250-460^{\circ} \mathrm{C}$. This weight loss 
TABLE 1: Variation of grain size, particle size, and strain and crystallinity index for PNZT system.

\begin{tabular}{|c|c|c|c|c|c|}
\hline \multirow[b]{2}{*}{ Composition } & \multirow[b]{2}{*}{$\begin{array}{l}\text { Grain size } \\
\quad(\mu \mathrm{m})\end{array}$} & \multicolumn{2}{|c|}{ Particle size (nm) } & \multirow[b]{2}{*}{$\begin{array}{l}\text { Strain } \\
(\varepsilon)\end{array}$} & \multirow[b]{2}{*}{$\begin{array}{l}\text { Crystallnity } \\
\text { index, } I_{\text {cry }}\end{array}$} \\
\hline & & $\begin{array}{l}\text { Debye Scherrer } \\
\text { method }\end{array}$ & $\begin{array}{l}\text { Williamson-hall } \\
\text { method }\end{array}$ & & \\
\hline $0.5 \mathrm{PN}-0.15 \mathrm{PZ}-0.35 \mathrm{PT}$ & 1.40 & 28.41 & 34.07 & 0.00229 & 49.27 \\
\hline $0.5 \mathrm{PN}-0.25 \mathrm{PZ}-0.25 \mathrm{PT}$ & 1.46 & 19.19 & 20.85 & 0.00035 & 76.08 \\
\hline $0.5 \mathrm{PN}-0.35 \mathrm{PZ}-0.15 \mathrm{PT}$ & 1.18 & 38.47 & 66.34 & 0.00108 & 30.67 \\
\hline
\end{tabular}

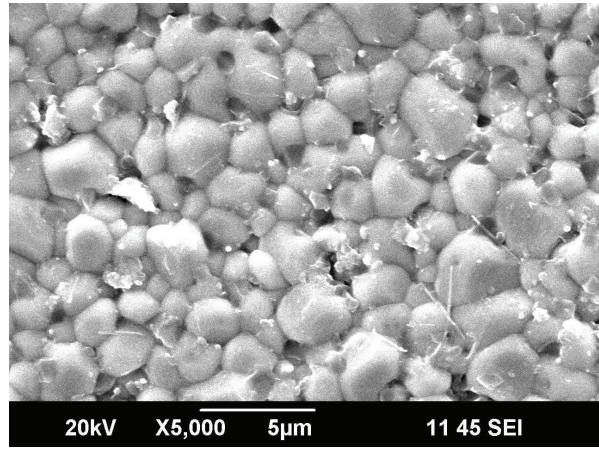

(a) $x=50, y=15 \%$

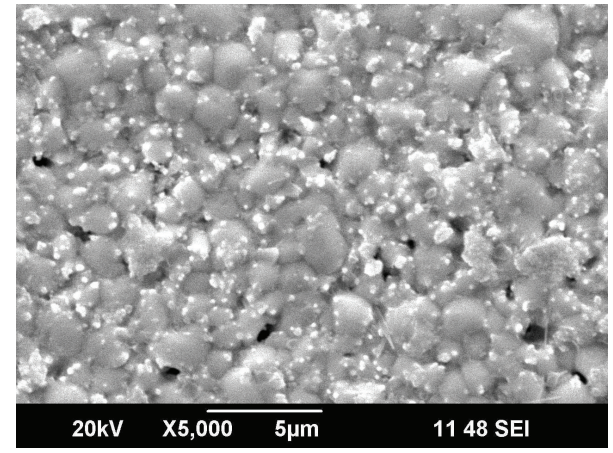

(b) $x=50, y=25 \%$

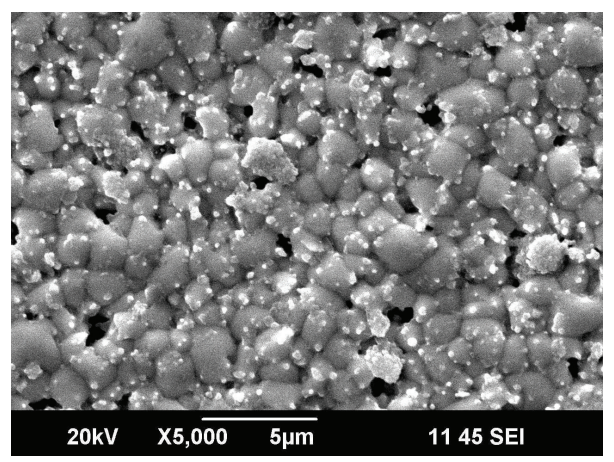

(c) $x=50, y=35 \%$

Figure 3: Scanning electron micrographs of $x \mathrm{PN}-y \mathrm{PZ}-(x-y) \mathrm{PT}$ (PNZT) composite $(x=50, y=15,25$, and 35\%).

may be attributed due to the presence of adsorbed and trapped solvent. Also, there is a sharp increase of weight loss between 325 and $375^{\circ} \mathrm{C}$ which is due to the reason that the solvent adsorbed on the outer surface of the particles requires relatively lower temperature to evaporate than those entrapped between the particles during milling. The weight loss at $578^{\circ} \mathrm{C}$ may be assigned due to loss of lead at high temperature suggesting that the precursor materials should be heated well at $600^{\circ} \mathrm{C}$ [33]. No significant weight loss was observed for temperature higher than $600^{\circ} \mathrm{C}$. The weight loss and the associated endothermic peak in DTA may be due to transformations into metastable phases, which formed prior to the crystallization of the perovskite phase. The last endothermic peak over the temperature range of $800-1000^{\circ} \mathrm{C}$ is not associated with weight loss in the TGA curve which corresponds to the crystallization of perovskite phase of lead niobate zirconate titanate (PNZT) [34].
3.3. Electrical Studies. Figures 6 and 7 show the dependence of real part of dielectric permittivity $\left(\varepsilon^{\prime}\right)$ and dielectric loss $(\tan \delta)$ on frequency at different temperatures. From the figures, the value of both $\varepsilon^{\prime}$ and $\tan \delta$ is found to decrease with increasing frequency which is a normal dielectric behaviour followed by all dielectric materials. In the lower frequency region, decrease in the value of $\varepsilon^{\prime}$ and $\tan \delta$ is observed which is due to the dominance of space charge polarization and interface effects at lower frequencies. However, in the high frequency region, frequency independent behaviour of these parameters is observed. At very low frequencies $(\omega \ll 1 / \tau)$, dipoles are able to follow the applied field and we have the value of dielectric constant at quasistatic field $\left(\varepsilon^{\prime} \sim \varepsilon_{s}\right)$. As the frequency increases, the dipoles began to lag behind the field resulting in a small increase in dielectric constant and as the characteristic frequency is reached, the value of dielectric constant drops which is the 


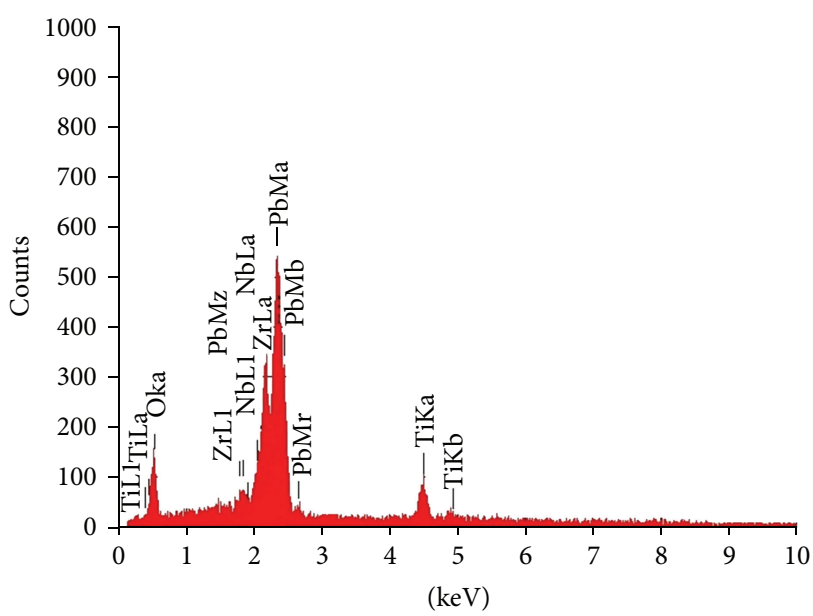

(a)

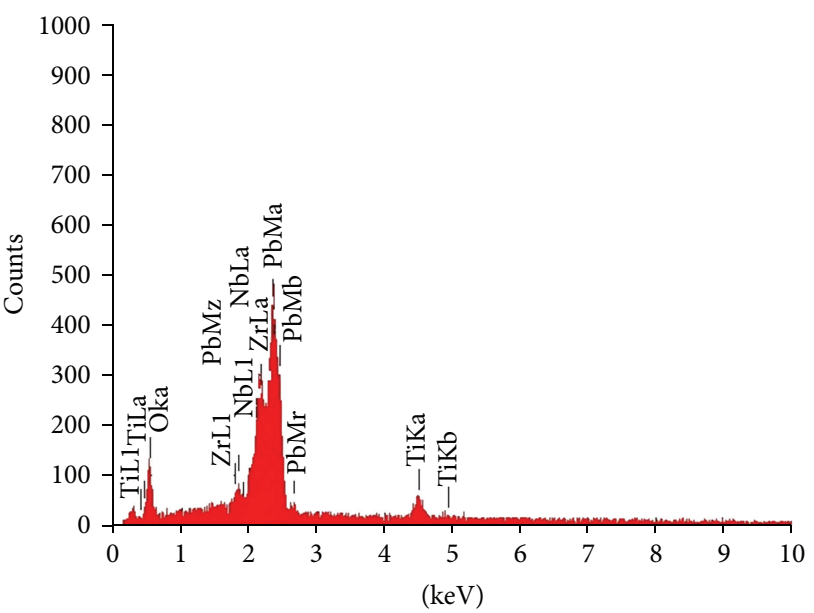

(b)

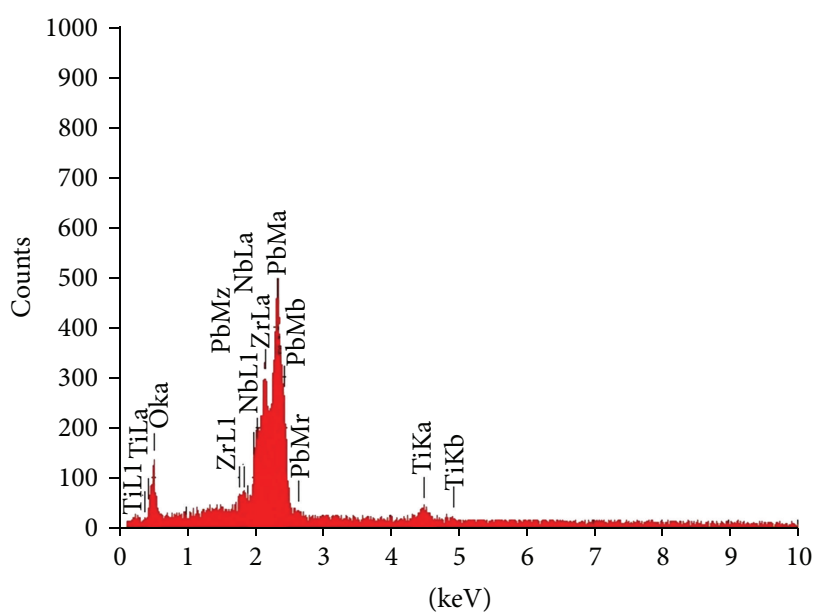

(c)

FIGURE 4: Energy dispersive X-ray analysis results for $x \mathrm{PN}-y \mathrm{PZ}-(x-y) \mathrm{PT}$ (PNZT) composite: (a) $x=50, y=15 \%$; (b) $x=50, y=25 \%$; and (c) $x=50, y=35 \%$.

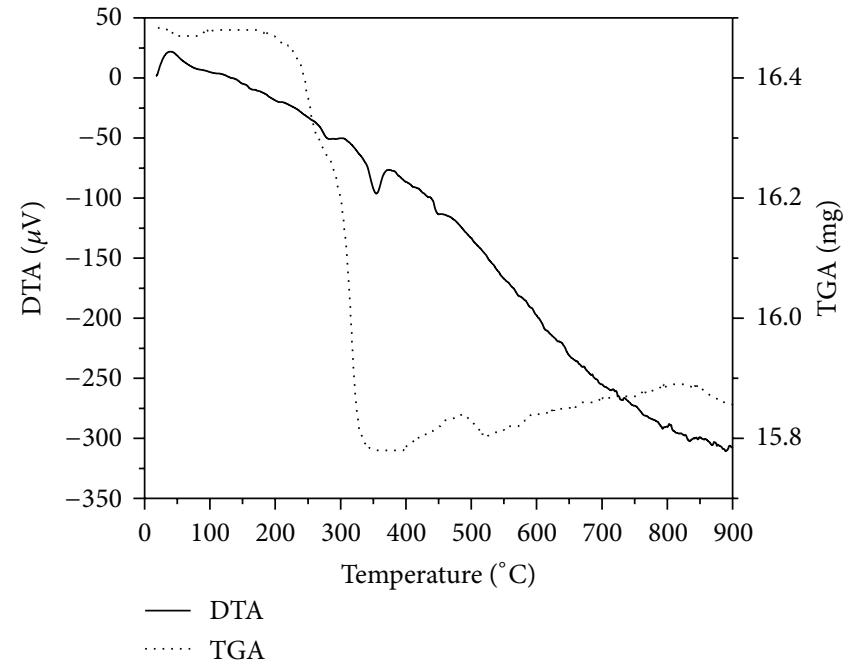

FIGURE 5: Thermogravimetric analysis along with differential thermal analysis (TGA/DTA) results for milled powder having composition 0.5PN-0.15PZ-0.35PT. 


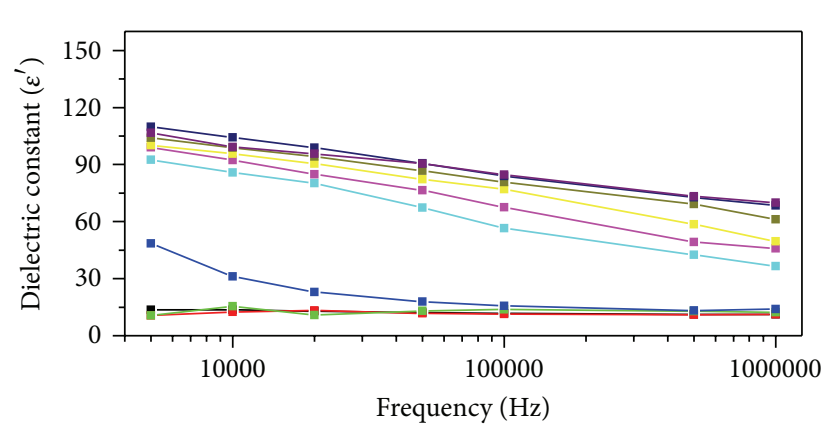

(a)

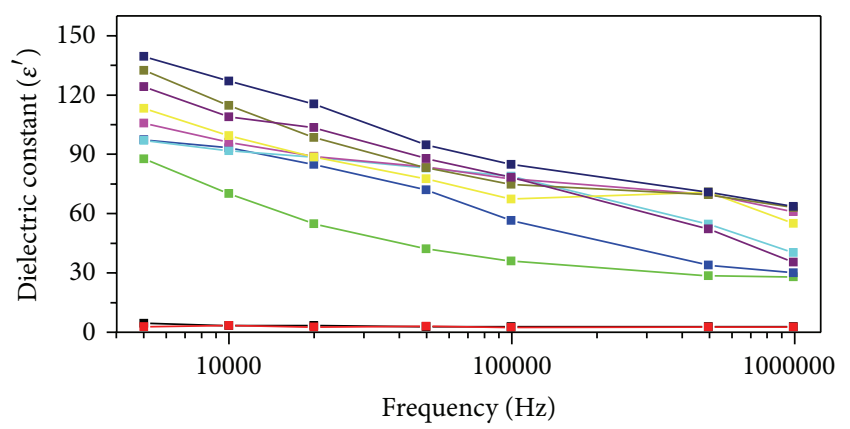

(b)

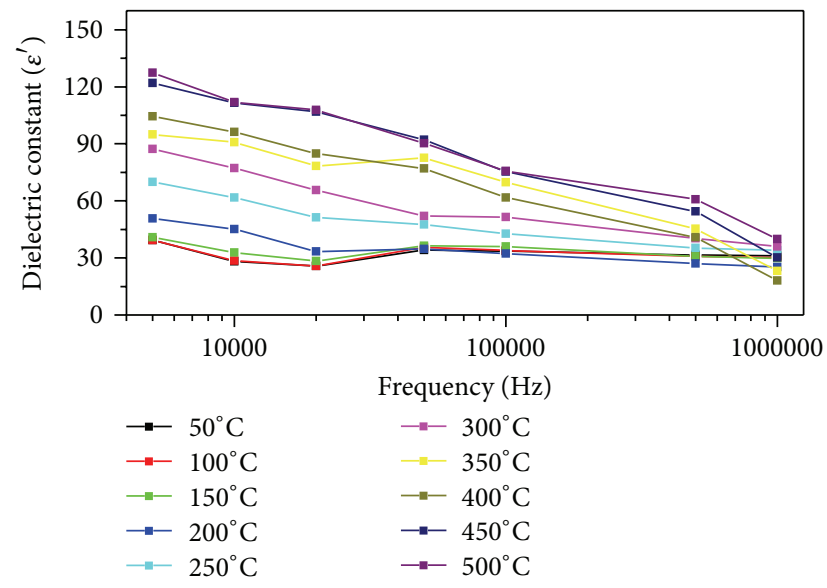

(c)

Figure 6: Variation of real part of dielectric constant with frequency for $x \mathrm{PN}-y \mathrm{PZ}-(x-y) \mathrm{PT}$ (a) $x=50, y=15 \%$; (b) $x=50, y=25 \%$; and (c) $x=50, y=35 \%$.

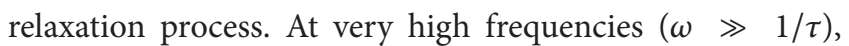
the dipoles are not able to align with the applied field and hence we have $\left(\varepsilon^{\prime} \sim \varepsilon_{\infty}\right)$. The maximum and minimum value of dielectric constant was found to be 111, 145, 128 and $11.7,0.65,37.4$ for $x=50, y=15,25$, and $35 \%$ in $x \mathrm{PN}-$ $y \mathrm{PZ}-(x-y) \mathrm{PT}$, respectively. The temperature dependence of dielectric constant $\left(\varepsilon^{\prime}\right.$, the real part of dielectric permittivity) at different frequencies is shown in Figure 8. Two types of dielectric anomalies were observed, one below $350^{\circ} \mathrm{C}$, which may be due to ferroelectric to antiferroelectric phase transition and another above $350^{\circ} \mathrm{C}$, which may be due to antiferroelectric to paraelectric phase transition. Thus, for these three compositions, two dielectric anomalies were seen which is of relaxor type. This relaxor-like anomaly was widely reported in various materials [35] and the anomaly in the temperature range of $400-900^{\circ} \mathrm{C}$ in oxide materials especially for those containing titanium is related to oxygen vacancies [36]. However, in present case, the formation of two much diffused peak at two different dielectric anomalies may be suggested to be due to the formation of mixed phase. Hence, in these three compositions because of poor peak formation, we are not able to give exact Curie temperature. At a given temperature other than the anomaly regions, the dielectric constant decreases with an increase in frequency and the dependence of dielectric constant with frequency increasingly varies with temperature. With increase in temperature, the peak position of dielectric constant in the curve shifts towards higher frequency side and this indicates frequency dispersion, thereby suggesting the thermally activated nature of dielectric relaxation. Further, all the plots show broad maxima, that is, diffuse phase transitions. In solid solutions, this broadening of peak or diffuse phase transition is a common feature due to the presence of more than one cation in the sublattice which produces some kind of heterogeneity. The poly-dispersive nature of dielectric relaxation can be analysed quantitatively through complex Argand plane plots of imaginary part of dielectric constant $\left(\varepsilon^{\prime \prime}\right)$ versus real part of dielectric constant $\left(\varepsilon^{\prime}\right)$ commonly called Cole-Cole plots. For a pure nondispersive Debye process, the plot is a perfect semicircle with its center located on the real axis. However, for poly-dispersive relaxation, the plot is a distorted semicircle with end points on the real axis and whose center lies below the real axis. Mathematically, the Cole-Cole plot obeys the following empirical relation [37]:

$$
\varepsilon^{*}=\varepsilon^{\prime}-j \varepsilon^{\prime \prime}=\varepsilon_{\infty}+\frac{\left(\varepsilon_{s}-\varepsilon_{\infty}\right)}{\left[1+(i \omega \tau)^{1-\alpha}\right]} .
$$

Here, " $\varepsilon_{\infty}$ " is the high frequency limit of the permittivity, " $\varepsilon_{s}$ " is the static dielectric constant, " $\varepsilon_{s}-\varepsilon_{\infty}$ " is the dielectric strength, " $\omega$ " is the angular frequency, " $\tau$ " is the mean relaxation time, and the parameter " $\alpha$ " represents the distribution 


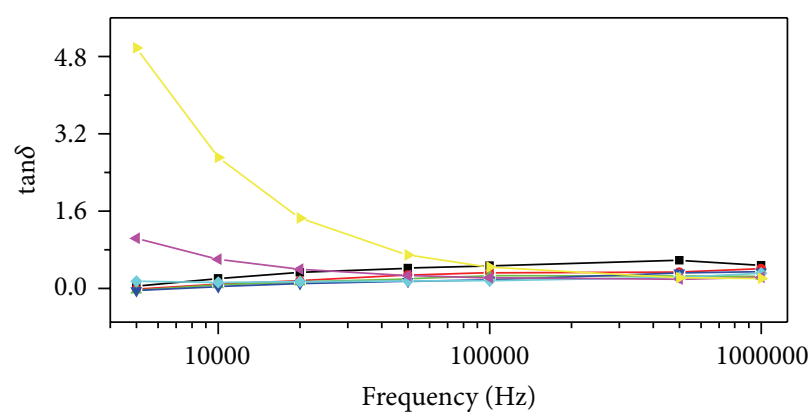

(a)

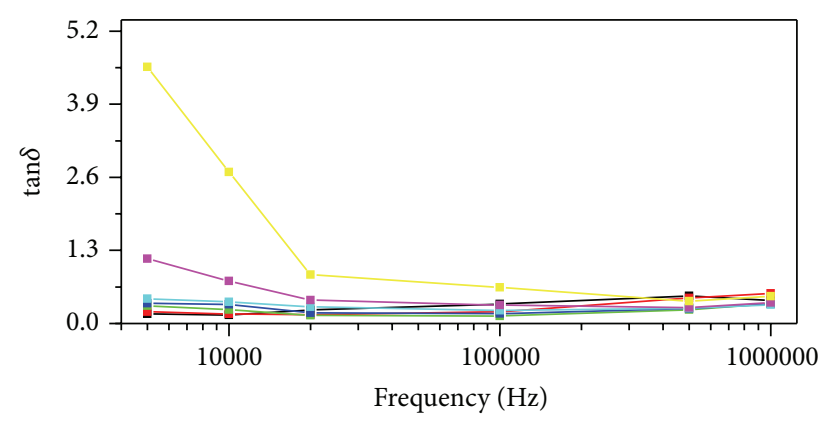

(b)

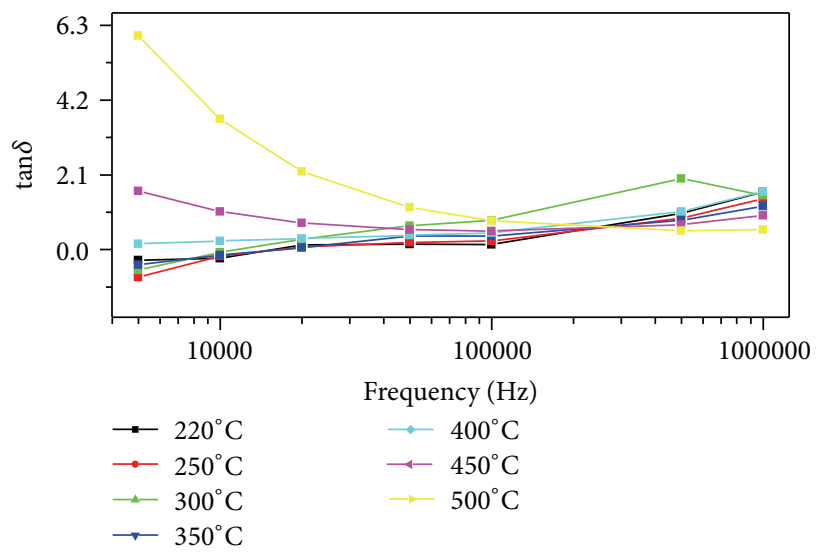

(c)

Figure 7: Variation of dielectric loss with frequency for $x \mathrm{PN}-y \mathrm{PZ}-(x-y) \mathrm{PT}$ (a) $x=50, y=15 \%$; (b) $x=50, y=25 \%$; and (c) $x=50, y=35 \%$.

of relaxation time and can be determined from the angle subtended by the radius of Cole-Cole plot with the real axis passing through the origin of the imaginary axis. At " $\varepsilon_{\infty}$ " and " $\varepsilon_{s}$ ", there will be no dielectric loss and maximum loss occurs at the midpoint between the two dielectric values. Figures 9(a)-9(c) depict the Cole-Cole plot representations at $T=$ $200^{\circ} \mathrm{C}$ for all the three compositions. At low temperatures, that is, less than $200^{\circ} \mathrm{C}$, the Cole-Cole plot resembles almost straight lines (not shown here) with large slopes, suggesting the insulating behaviour at low temperatures. However, with increasing temperature, the slopes of these curves decrease and they bend towards the real axis forming semicircles suggesting an increase in conductivity of the material with temperature. The formation of two semicircular arcs in the Cole-Cole plot can be explained on the basis of two parallel RC elements connected in series, one associated with grain (higher frequency range) and the other with grain boundary (lower frequency range) as the relaxation time for the grain boundary is much larger than that of the bulk crystal, thereby indicating negative temperature coefficient of resistance (NCTR) behaviour of PNZT like that of a semiconducting material [38]. As " $\varepsilon$ " is directly related to capacitance, that is, $\varepsilon^{\prime}=C t / \varepsilon_{0} A$, where " $A$ " is area in $\mathrm{mm}^{2}$ and " $t$ " is thickness of sample in $\mathrm{mm}$, the capacitances $C_{g}$ (associated with grain) and $C_{\mathrm{gb}}$ (associated with grain boundary) have been calculated from the low frequency intercept of the semicircles on the real $\varepsilon^{\prime}$-axis. As relaxation
TABLE 2: Values of grain resistance $\left(R_{g}\right)$, grain capacitance $\left(C_{g}\right)$, grain boundary resistance $\left(R_{\mathrm{gb}}\right)$, and grain boundary capacitance $\left(C_{\mathrm{gb}}\right)$ obtained from Cole-Cole plot for PNZT system.

\begin{tabular}{lcccc}
\hline Composition & $R_{g} \times 10^{4}$ & $C_{g}(\mathrm{pF})$ & $R_{\mathrm{gb}} \times 10^{5}$ & $C_{\mathrm{gb}}(\mathrm{pF})$ \\
\hline $0.5 \mathrm{PN}-0.15 \mathrm{PZ}-0.35 \mathrm{PT}$ & 42.3 & 23.61 & 24.8 & 40.26 \\
$0.5 \mathrm{PN}-0.25 \mathrm{PZ}-0.25 \mathrm{PT}$ & 30.0 & 59.54 & 68.5 & 29.21 \\
$0.5 \mathrm{PN}-0.35 \mathrm{PZ}-0.15 \mathrm{PT}$ & 65.1 & 5.12 & 44.5 & 14.97 \\
\hline
\end{tabular}

time, $\tau_{g}=R_{g} C_{g}$ and $\tau_{\mathrm{gb}}=R_{\mathrm{gb}} C_{\mathrm{gb}}$, the resistances $R_{g}$ and $R_{\mathrm{gb}}$ were obtained from the maximum condition of the semicircles, that is, $\omega_{\operatorname{mac}}=1 / \tau=2 \pi f_{\max }$, where " $f_{\max }$ " is the frequency at the maximum of semicircle. The values of $R_{g}$, $R_{\mathrm{gb}}, C_{g}$, and $C_{\mathrm{gb}}$ obtained from the Cole-Cole plot are given in Table 2. As seen from table, bulk resistance decreases with increase in grain size. Also, " $\alpha$ " lies below the real axis and its value was found to be $0.42,0.37$, and 0.16 radians for the three compositions, thereby confirming the non-Debye type relaxation in PNZT.

3.4. ac Conductivity Analysis. ac conductivity $\left(\sigma_{\mathrm{ac}}\right)$ has been calculated from the dielectric loss $(\tan \delta)$ data using the empirical formula [39]:

$$
\sigma_{\mathrm{ac}}=\omega \varepsilon_{0} \varepsilon^{\prime} \tan \delta=2 \pi f \varepsilon_{0} \varepsilon^{\prime} \tan \delta,
$$




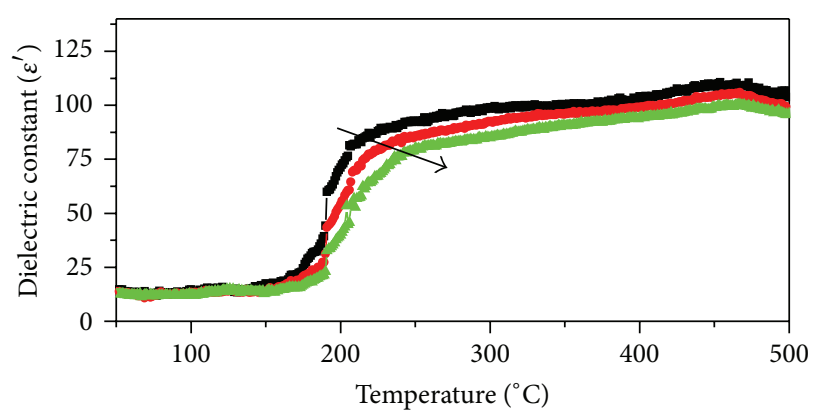

(a)

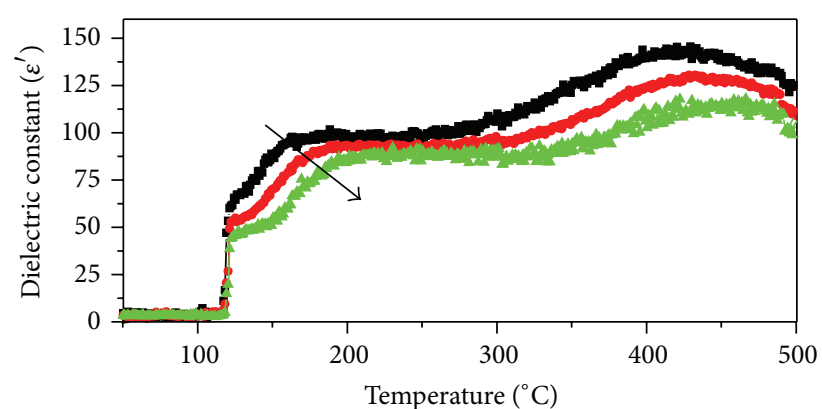

(b)

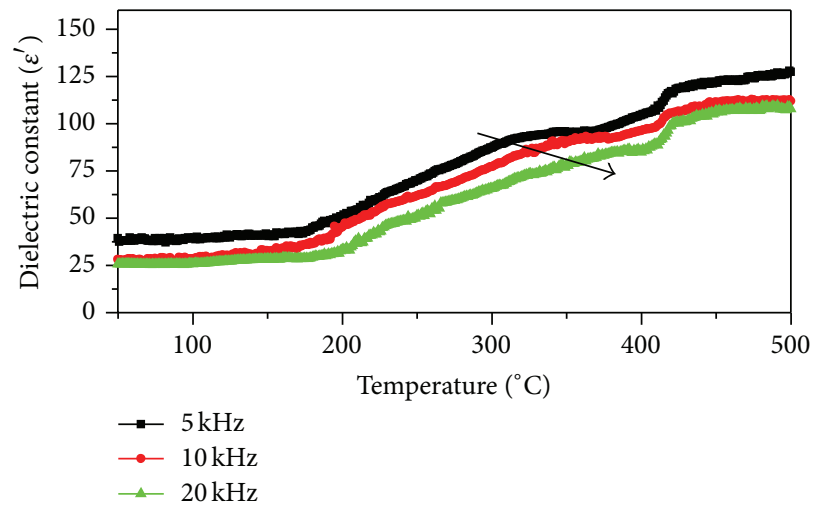

(c)

FIGURE 8: Variation of real part of dielectric constant with temperature at 5, 10, and $20 \mathrm{kHz}$ frequency for $x \mathrm{PN}-y \mathrm{PZ}-(x-y) \mathrm{PT}(\mathrm{a}) x=50, y=$ $15 \%$; (b) $x=50, y=25 \%$; and (c) $x=50, y=35 \%$.

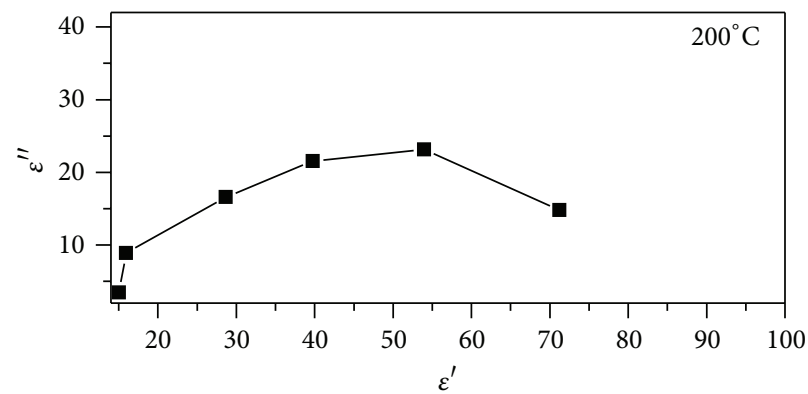

(a)

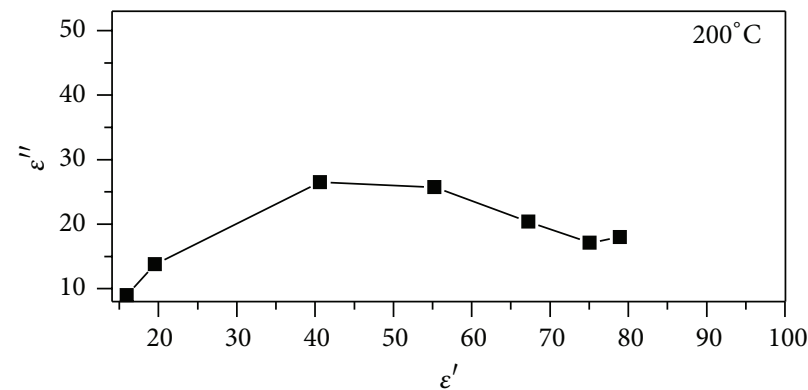

(b)

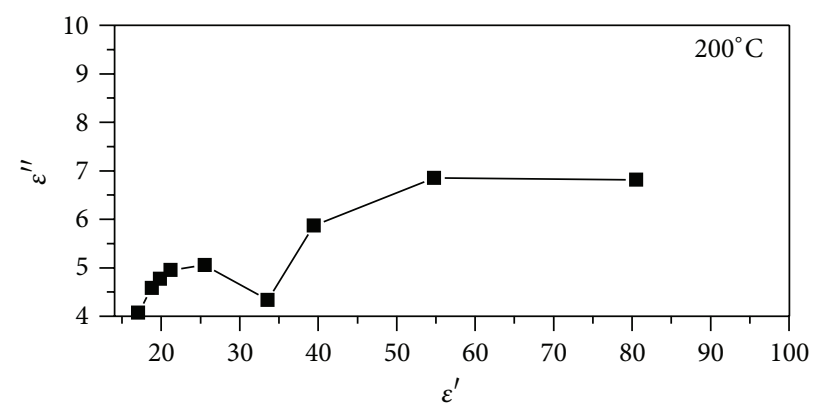

(c)

FIGURE 9: Complex plane Argand plot between $\varepsilon^{\prime \prime}$ and $\varepsilon^{\prime}$ at $200^{\circ} \mathrm{C}$ for $x \mathrm{PN}-y \mathrm{PZ}-(x-y) \mathrm{PT}$ (a) $x=50, y=15 \%$; (b) $x=50, y=25 \%$; and (c) $x$ $=50, y=35 \%$. 
TABLE 3: Variation of activation energy $\left(E_{a}\right)$ with frequency and exponent $(s)$ with temperature for three different compositions of PNZT system.

\begin{tabular}{|c|c|c|c|c|c|c|c|c|c|}
\hline \multirow{2}{*}{ Composition } & \multicolumn{3}{|c|}{ Activation energy $\left(E_{a}\right)$ for different frequencies $(\mathrm{eV})$} & \multicolumn{6}{|c|}{ Exponent $(s)$ for different temperatures $\left({ }^{\circ} \mathrm{C}\right)$} \\
\hline & $5 \mathrm{kHz}$ & $10 \mathrm{kHz}$ & $20 \mathrm{kHz}$ & 250 & 300 & 350 & 400 & 450 & 500 \\
\hline $0.5 \mathrm{PN}-0.15 \mathrm{PZ}-0.35 \mathrm{PT}$ & 0.66 & 0.46 & 0.27 & 1.0 & 1.1 & 1.3 & 1.0 & 0.7 & 0.3 \\
\hline $0.5 \mathrm{PN}-0.25 \mathrm{PZ}-0.25 \mathrm{PT}$ & 0.24 & 0.16 & 0.13 & 1.0 & 0.9 & 0.8 & 0.8 & 0.6 & 0.3 \\
\hline $0.5 \mathrm{PN}-0.35 \mathrm{PZ}-0.15 \mathrm{PT}$ & 0.99 & 0.50 & 0.23 & 0.8 & 1.2 & 1.0 & 0.8 & 0.4 & 0.1 \\
\hline
\end{tabular}

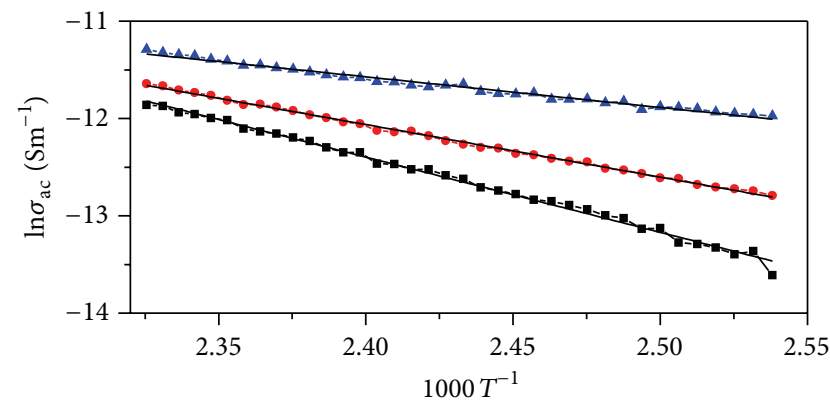

(a)

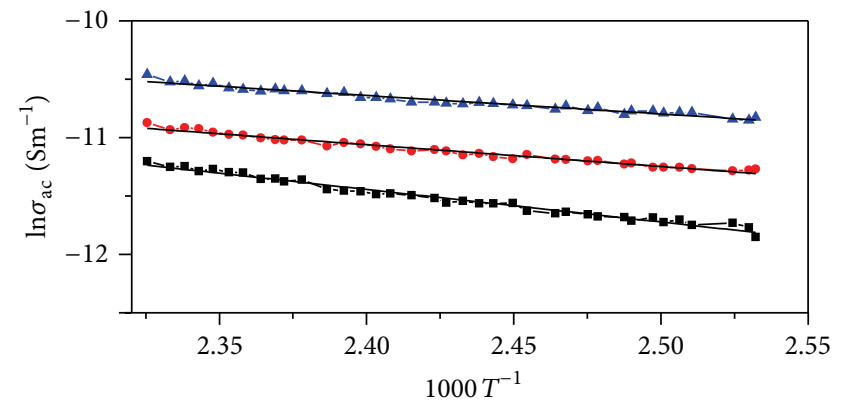

(b)

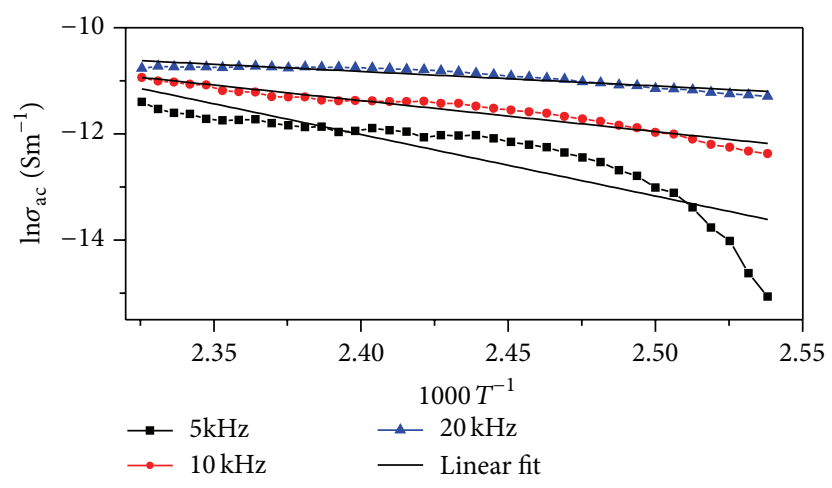

(c)

Figure 10: Temperature dependence of the ac conductivity at 5, 10, and $20 \mathrm{kHz}$ frequency for $x \mathrm{PN}-y \mathrm{PZ}-(x-y) \mathrm{PT}$ (a) $x=50, y=15 \%$; (b) $x=50, y=25 \%$; and (c) $x=50, y=35 \%$.

where $\varepsilon_{0}=8.845 \times 10^{-12} \mathrm{Fm}^{-1}$ is the permittivity of free space. Figure 10 shows the variation of ac conductivity as a function of $1000 / T$. The results indicate an increase in conductivity with rise in temperature, thereby indicating the negative temperature coefficient of resistance (NTCR) character. This type of temperature dependence indicates that the electrical conduction in this material is the thermally activated transport process governed by Arrhenius equation [29]:

$$
\sigma_{\mathrm{ac}}=\sigma_{0} \exp \left(-\frac{E_{a}}{k T}\right),
$$

where " $\sigma_{0}$ " is the ac conductivity preexponential factor and " $E_{a}$ " the ac conductivity activation energy. The activation energy of these three compositions in the temperature range of $394-430^{\circ} \mathrm{C}$ was calculated from the slope of $\ln \left(\sigma_{\mathrm{ac}}\right)$ versus $1000 / T$ graph and is given in Table 3 . As seen from the table, the activation energy shows a decreasing trend with increase in frequency. This is because of the reason that the increase in the frequency of the applied field results in the enhancement of the charge carriers to jump between localized states which therefore results in the decrease in electrical activation energy with increasing frequency $[40,41]$. The low value of activation energy may be due to the carrier transport through hopping between localized states in a disordered manner [42, 43]. Also, the activation energy is found to be minimum for the $0.5 \mathrm{PN}-0.25 \mathrm{PZ}-0.25 \mathrm{PT}$ composition thereby suggesting the decrease in resistivity. The frequency dependence of ac conductivity follows the universal power law $[44,45]$, that is,

$$
\sigma(\omega)=A \omega^{s},
$$

where " $A$ " is temperature dependent constant, " $\omega$ " is the angular frequency of the applied ac field, and the exponent " $s$ " is temperature as well as frequency dependent constant and its value approaches unity at low temperature and decreases with increase in temperature. The values of " $s$ " deduced from the slopes of $\ln \left(\sigma_{\mathrm{ac}}\right)$ versus $\ln (\omega)$ curves are given in Table 3 , from which one can see that " $s$ " is approaching 


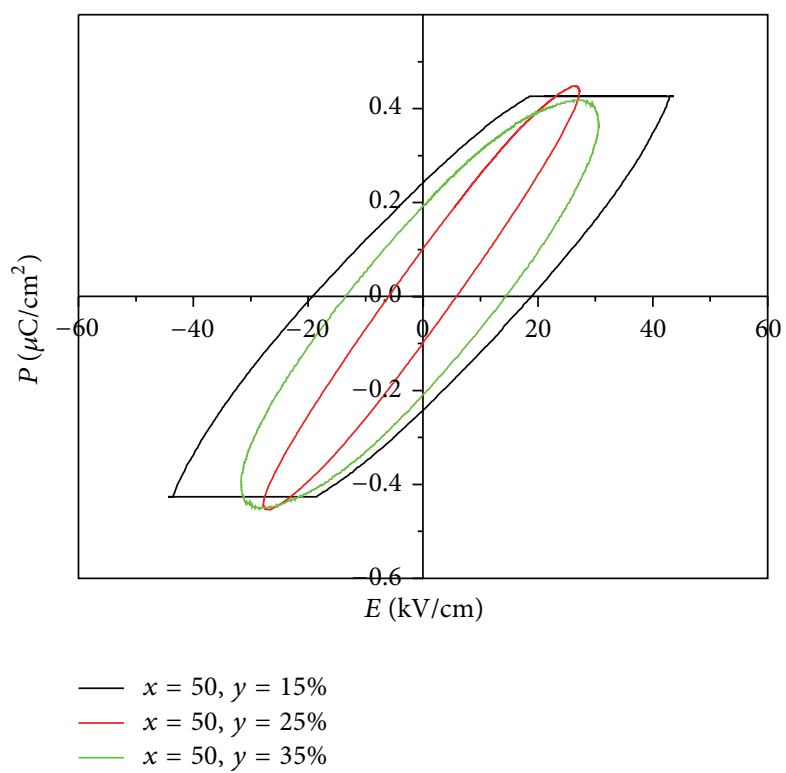

FIGURE 11: Variation of polarization with electric field for $x \mathrm{PN}-y \mathrm{PZ}-(x-y) \mathrm{PT}(x=50, y=15,25$, and 35\%) at room temperature.

unity at low temperature which confirms assumption that ac conductivity is predominant due to the relaxation dipole moment. The power law dependence of the ac conductivity on frequency corresponds to the short range hopping of carriers through trap sites separated by an energy barrier of various heights. Since the value of " $s$ " actually scales the extent of localized charge carriers [45], for example, in the case of $s=$ 0 , the above equation shows the usual reciprocal frequency behaviour, and the system is nondispersive transport of free charge carriers process; for $s=1$, the system has the feature of nearly constant loss relating to strictly localized carriers, while for $0<s<1$, the system obeys the universal power law with confined hopping carriers. It, therefore, follows that the relaxing species for the low-temperature anomaly are confined carriers and for the high-temperature anomaly are free ones.

3.5. Ferroelectric Studies. The polarization versus electric field (P-E) hysteresis loops with different $\mathrm{Zr}$ concentrations are shown in Figure 11 whereas the values of remanent polarization and coercivity are given in Table 4 . The minimum value of remnant polarization, $P_{r} \sim 0.10 \mu \mathrm{C} / \mathrm{cm}^{2}$, and the coercive field, $E_{c} \sim 6.33 \mathrm{kV} / \mathrm{cm}$, was observed for the composition $0.5 \mathrm{PN}-0.25 \mathrm{PZ}-0.25 \mathrm{PT}$. The saturation polarization was obtained only in the case of $0.5 \mathrm{PN}-0.15 \mathrm{PZ}-$ $0.35 \mathrm{PT}$ with a value of $P_{s}=0.43 \mu \mathrm{C} / \mathrm{cm}^{2}$ at a maximum applied field of $20 \mathrm{kV} / \mathrm{cm}$. However, in the other two cases, no saturation in polarization in P-E curve was achieved up to the maximum applied field which is due to the low resistivity of the composition [38]. The compositions with 25 and 35\% Zr content could not withstand the electric field beyond 14 and $16 \mathrm{kV} / \mathrm{cm}$ and further increase in electric field led to the electrical breakdown and thus unsaturated P-E loops were obtained in these two compositions. Low value of coercivity and remanent polarization suggests that these ternary systems are soft ferroelectric.
TABLE 4: Variation of remnant polarization $\left(P_{r}\right)$ and coercivity $\left(E_{c}\right)$ for PNZT ternary system.

\begin{tabular}{lcc}
\hline Composition & $\begin{array}{c}\text { Remnant } \\
\text { polarization } \\
\left(P_{r}\right)\left(\mu \mathrm{C} / \mathrm{cm}^{2}\right)\end{array}$ & $\begin{array}{c}\text { Coercivity } \\
\left(E_{c}\right) \\
(\mathrm{kV} / \mathrm{cm})\end{array}$ \\
\hline $0.5 \mathrm{PN}-0.15 \mathrm{PZ}-0.35 \mathrm{PT}$ & 0.24 & 19.15 \\
$0.5 \mathrm{PN}-0.25 \mathrm{PZ}-0.25 \mathrm{PT}$ & 0.10 & 6.33 \\
$0.5 \mathrm{PN}-0.35 \mathrm{PZ}-0.15 \mathrm{PT}$ & 0.20 & 13.61 \\
\hline
\end{tabular}

\section{Conclusions}

Polycrystalline compositions of $x \mathrm{PN}-y \mathrm{PZ}-(x-y) \mathrm{PT}$ (where $x=0.5, y=0.15,0.25$, and 0.35$)$ known as PNZT ceramic were prepared by conventional mixed oxide route. Room temperature X-ray diffraction analysis of PNZT confirms the well-crystallized rhombohedral phase of the prepared compositions with the presence of both PN and PZT phase. Scanning electron micrographs depict formation of fairly dense grains with average grain size in the submicrometric range whereas presence of major elements was confirmed by energy dispersive X-ray analysis (EDAX). Thermal analysis of the milled powder suggests the optimum temperature for calcination is $900^{\circ} \mathrm{C}$. Electrical properties carried by dielectric spectroscopy on PNZT indicate that the material exhibits two types of dielectric anomalies, one below $350^{\circ} \mathrm{C}$ and another above $350^{\circ} \mathrm{C}$ and shifting of maximum value of dielectric constant towards higher temperature side with increase in frequency indicating the relaxor behaviour. The temperature dependence of ac conductivity indicates increase in conductivity with rise in temperature thereby suggesting negative temperature coefficient of resistance (NCTR) character. The poly-dispersive nature of dielectric relaxation has been studied by complex plane Argand plot and its shape indicates the presence of both grain and grain boundary 
effect in the relaxation process in PNZT. The frequency dependence of ac conductivity obeys the universal power law. The ferroelectric studies suggest these ternary systems to be soft ferroelectric.

\section{Conflict of Interests}

The authors declare that there is no conflict of interests regarding publication of this paper.

\section{Acknowledgments}

The authors express their gratitude to Sophisticated Test and Instrumentation Centre (STIC), Cochin University, for providing XRD and SEM-EDAX facilities. They are also thankful to Dr. Binay Kumar, Department of Physics, University of Delhi, for providing the facility of P-E loop tracer.

\section{References}

[1] M. M. Vijatović, J. D. Bobić, and B. D. Stojanović, "History and challenges of barium titanate: part II," Science of Sintering, vol. 40, no. 3, pp. 235-244, 2008.

[2] N. Vittayakorn, G. Rujijanagul, X. Tan, M. A. Marquardt, and D. P. Cann, "The morphotropic phase boundary and dielectric properties of the $\mathrm{xb}\left(\mathrm{Zr}_{1 / 2} \mathrm{Ti}_{1 / 2}\right) \mathrm{O}_{3}-(1-\mathrm{x}) \mathrm{Pb}\left(\mathrm{Ni}_{1 / 3} \mathrm{Nb}_{2 / 3}\right) \mathrm{O}_{3}$ perovskite solid solution," Journal of Applied Physics, vol. 96, no. 9, pp. 5103-5109, 2004.

[3] L. E. Cross, "Relaxor ferroelectrics: an overview," Ferroelectrics, vol. 151, no. 1, pp. 305-320, 1994.

[4] S.-E. Park, "Relaxor based ferroelectric single crystals for electro-mechanical actuators," Materials Research Innovations, vol. 1, no. 1, pp. 20-25, 1997.

[5] G. Goodman, "Ferroelectric properties of lead metaniobate," Journal of the American Ceramic Society, vol. 36, no. 11, pp. 368372, 1953.

[6] M. H. Francombe and B. Lewis, "Structural, dielectric and optical properties of ferroelectric leadmetaniobate," Acta Crystallographica, vol. 11, no. 10, pp. 696-703, 1958.

[7] E. C. Subbarao and G. Shirane, "Nonstoichiometry and ferroelectric properties of $\mathrm{PbNb}_{2} \mathrm{O}_{6}$-type compounds," The Journal of Chemical Physics, vol. 32, no. 6, pp. 1846-1851, 1960.

[8] L. A. Reznichenko, G. A. Geguzina, and N. V. Dergunova, "Piezoelectric solid solutions based on alkali niobates," Inorganic Materials, vol. 34, no. 2, pp. 167-173, 1998.

[9] I. P. Raevski and S. A. Prosandeev, "A new, lead free, family of perovskites with a diffuse phase transition: $\mathrm{NaNbO}_{3}$-based solid solutions," Journal of Physics and Chemistry of Solids, vol. 63, no. 10, pp. 1939-1950, 2002.

[10] J. M. Haussonne, G. Desgardin, A. Herve, and B. Boufrou, "Dielectric ceramics with relaxors and a tetragonal tungsten bronze," Journal of the European Ceramic Society, vol. 10, no. 6, pp. 437-452, 1992.

[11] M. Venet, A. Vendramini, F. L. Zabotto, F. Guerrero, D. Garcia, and J. A. Eiras, "Piezoelectric properties of undoped and titanium or barium-doped lead metaniobate ceramics," Journal of the European Ceramic Society, vol. 25, no. 12, pp. 2443-2446, 2005.

[12] K. R. Sahu and U. De, "Thermal characterization of piezoelectric and non-piezoelectric Lead Meta-Niobate," Thermochimica Acta, vol. 490, no. 1-2, pp. 75-77, 2009.
[13] U. De, K. R. Sahu, K. R. Chakraborty, and S. K. Pratihar, "Dielectric and thermal investigations on $\mathrm{PbNb}_{2} \mathrm{O}_{6}$ in pure piezoelectric phase and pure non-piezoelectric phase," Integrated Ferroelectrics, vol. 119, no. 1, pp. 96-109, 2010.

[14] K. R. Sahu and U. De, "Dielectric properties of $\mathrm{PbNb}_{2} \mathrm{O}_{6}$ up to $700^{\circ} \mathrm{C}$ from impedance spectroscopy," Journal of Materials, vol. 2013, Article ID 702946, 15 pages, 2013.

[15] K. R. Sahu and U. De, "Dielectric properties of rhombohedral $\mathrm{PbNb}_{2} \mathrm{O}_{6}$," Journal of Solid State Physics, vol. 2013, Article ID 451563, 9 pages, 2013.

[16] Y. Xu, Ferroelectric Materials and Their Applications, Elsevier Science, Amsterdam, The Netherlands, 1991.

[17] G. H. Haertling, "Ferroelectric ceramics: history and technology," Journal of the American Ceramic Society, vol. 82, no. 4, pp. 797-818, 1999.

[18] X. Dai and Y. Wang, "Multistage heat-electric energy conversion working on $\mathrm{F}_{R(L T)}-\mathrm{F}_{R(H T)}$ phase transition in NB doped PZT97/3 ceramics," Ferroelectrics, vol. 109, no. 1, pp. 253-258, 1990.

[19] N. Duan, N. Cereceda, B. Noheda, and J. A. Gonzalo, "Dielectric characterization of the phase transitions in $\mathrm{Pb}_{1-y / 2}\left(\mathrm{Zr}_{1-x} \mathrm{Ti}_{x}\right)_{1-y} \mathrm{Nb}_{y} \mathrm{O}_{3}(0.03<x<0.04,0.02<y<0.05)$," Journal of Applied Physics, vol. 82, article 779, 1997.

[20] Z. Ujma, J. Handerek, and G. E. Kugel, "Phase transitions in $\mathrm{Nb}$-doped $\mathrm{Pb}\left(\mathrm{Zr}_{0.95} \mathrm{Ti}_{0.05}\right) \mathrm{O}_{3}$ ceramics investigated by dielectric, pyroelectric and Raman scattering measurements," Ferroelectrics, vol. 198, no. 1, pp. 77-97, 1997.

[21] H. C. Nie, X. L. Dong, N. B. Feng et al., "Quantitative dependence of the properties of $\mathrm{Pb}_{0.99}\left(\mathrm{Zr}_{0.95} \mathrm{Ti}_{0.05}\right)_{0.98} \mathrm{Nb}_{0.02} \mathrm{O}_{3}$ ferroelectric ceramics on porosity," Materials Research Bulletin, vol. 45, no. 5, pp. 564-567, 2010.

[22] R. D. Klissurska, K. G. Brooks, I. M. Reaney, C. Pawlaczyk, M. Kosec, and N. Setter, "Effect of Nb doping on the microstructure of sol-gel-derived PZT thin films," Journal of the American Ceramic Society, vol. 78, no. 6, pp. 1513-1520, 1995.

[23] V. Kayasu and M. Ozenbas, "The effect of Nb doping on dielectric and ferroelectric properties of PZT thin films prepared by solution deposition," Journal of the European Ceramic Society, vol. 29, no. 6, pp. 1157-1163, 2009.

[24] H. Han, S. Kotru, J. Zhong, and R. K. Pandey, "Effect of Nb doping on pyroelectric property of lead zirconate titanate films prepared by chemical solution deposition," Infrared Physics and Technology, vol. 51, no. 3, pp. 216-220, 2008.

[25] D. F. Ryder and N. K. Raman, "Sol-Gel processing of Nb-doped $\mathrm{Pb}(\mathrm{Zr}, \mathrm{Ti}) \mathrm{O}_{3}$ thin films for ferroelectric memory applications," Journal of Electronic Materials, vol. 21, no. 10, pp. 971-975, 1992.

[26] R. Gerson, "Variation in ferroelectric characteristics of lead zirconate titanate ceramics due to minor chemical modifications," Journal of Applied Physics, vol. 31, no. 1, pp. 188-194, 1960.

[27] R. Gerson and H. Jaffe, "Electrical conductivity in lead titanate zirconate ceramics," Journal of Physics and Chemistry of Solids, vol. 24, no. 8, pp. 979-984, 1963.

[28] S. K. Hau, K. H. Wong, P. W. Chan, and C. L. Choy, "Intrinsic resputtering in pulsed-laser deposition of lead-zirconatetitanate thin films," Applied Physics Letters, vol. 66, pp. 245-247, 1995.

[29] K. R. Chakraborty, K. R. Sahu, A. De, and U. De, "Structural characterization of orthorhombic and rhombohedral lead meta-niobate samples," Integrated Ferroelectrics, vol. 120, no. 1, pp. 102-113, 2010. 
[30] B. D. Cullity and S. R. Stock, Elements of X-Ray Diffraction, Prentice Hall, Englewood Cliffs, NJ, USA, 3rd edition, 2001.

[31] V. D. Mote, Y. Purushotham, and B. N. Dole, "WilliamsonHall analysis in estimation of lattice strain in nanometer-sized ZnO particles," Journal of Theoretical and Applied Physics, vol. 6, article 6, 2012.

[32] M. N. Rifaya, T. Theivasanthi, and M. Alagar, "Chemical cappingsynthesis of nickel oxide nanoparticles and their characterization studies," Nanoscience and Nanotechnology, vol. 2, no. 5, pp. 134-138, 2012.

[33] A. Shukla and R. N. P. Choudhary, "Ferroelectric phasetransition and conductivity analysis of $\mathrm{La}^{3+} / \mathrm{Mn}^{4+}$ modified $\mathrm{PbTiO}_{3}$ nanoceramics," Physica B: Condensed Matter, vol. 405, no. 11, pp. 2508-2515, 2010.

[34] B. Praveenkumar, H. H. Kumar, D. K. Kharat, and B. S. Murty, "Investigation and characterization of La-doped PZT nanocrystalline ceramic prepared by mechanical activation route," Materials Chemistry and Physics, vol. 112, no. 1, pp. 3134, 2008.

[35] C. C. Wang and S. X. Dou, "Pseudo-relaxor behaviour induced by Maxwell-Wagner relaxation," Solid State Communications, vol. 149, no. 45-46, pp. 2017-2020, 2009.

[36] C. Ang, Z. Yu, and L. E. Cross, “Oxygen-vacancy-related lowfrequency dielectric relaxation and electrical conduction in $\mathrm{Bi}: \mathrm{SrTiO}_{3}$," Physical Review B-Condensed Matter and Materials Physics, vol. 62, no. 1, pp. 228-236, 2000.

[37] K. S. Cole and R. H. Cole, "Dispersion and absorption in dielectrics I. Alternating current characteristics," The Journal of Chemical Physics, vol. 9, no. 4, pp. 341-351, 1941.

[38] A. K. Roy, A. Singh, K. Kumari, K. A. Nath, A. Prasad, and K. Prasad, "Electrical properties and AC conductivity of $\left(\mathrm{Bi}_{0.5} \mathrm{Na}_{0.5}\right)_{0.94} \mathrm{Ba}_{0.06} \mathrm{TiO}_{3}$ ceramic," ISRN Ceramics, vol. 2012, Article ID 854831, 10 pages, 2012.

[39] R. N. P. Choudhary, D. K. Pradhan, G. E. Bonilla, and R. S. Katiyar, "Effect of La-substitution on structural and dielectric properties of $\mathrm{Bi}\left(\mathrm{Sc}_{1} /{ }_{2} \mathrm{Fe}_{1 / 2}\right) \mathrm{O}_{3}$ ceramics," Journal of Alloys and Compounds, vol. 437, no. 1-2, pp. 220-224, 2007.

[40] A. A. Ebnalwaled, "On the conduction mechanism of p-type GaSb bulk crystal," Materials Science and Engineering B: SolidState Materials for Advanced Technology, vol. 174, no. 1-3, pp. 285-289, 2010.

[41] N. A. Hegab, M. A. Afifi, H. E. Atyia, and A. S. Farid, "AC conductivity and dielectric properties of amorphous $\mathrm{Se}_{80} \mathrm{Te}_{20-x} \mathrm{Ge}_{x}$ chalcogenide glass film compositions," Journal of Alloys and Compounds, vol. 477, no. 1-2, pp. 925-930, 2009.

[42] S. Upadhyay, A. K. Sahu, D. Kumar, and O. Parkash, "Probing electrical conduction behavior of $\mathrm{BaSnO}_{3}$," Journal of Applied Physics, vol. 84, no. 2, pp. 828-832, 1998.

[43] K. Prasad, C. K. Suman, and R. N. P. Choudhary, "Electrical characterisation of $\mathrm{Pb}_{2} \mathrm{Bi}_{3} \mathrm{SmTi}_{5} \mathrm{O}_{18}$ ceramic using impedance spectroscopy," Advances in Applied Ceramics, vol. 105, no. 5, pp. 258-264, 2006.

[44] A. G. Hunt, "Ac hopping conduction: perspective from percolation theory," Philosophical Magazine B, vol. 81, no. 9, pp. 875913, 2001.

[45] A. R. Long, Hopping Transport in Solid, North-Holland, Amsterdam, The Netherlands, 1991. 

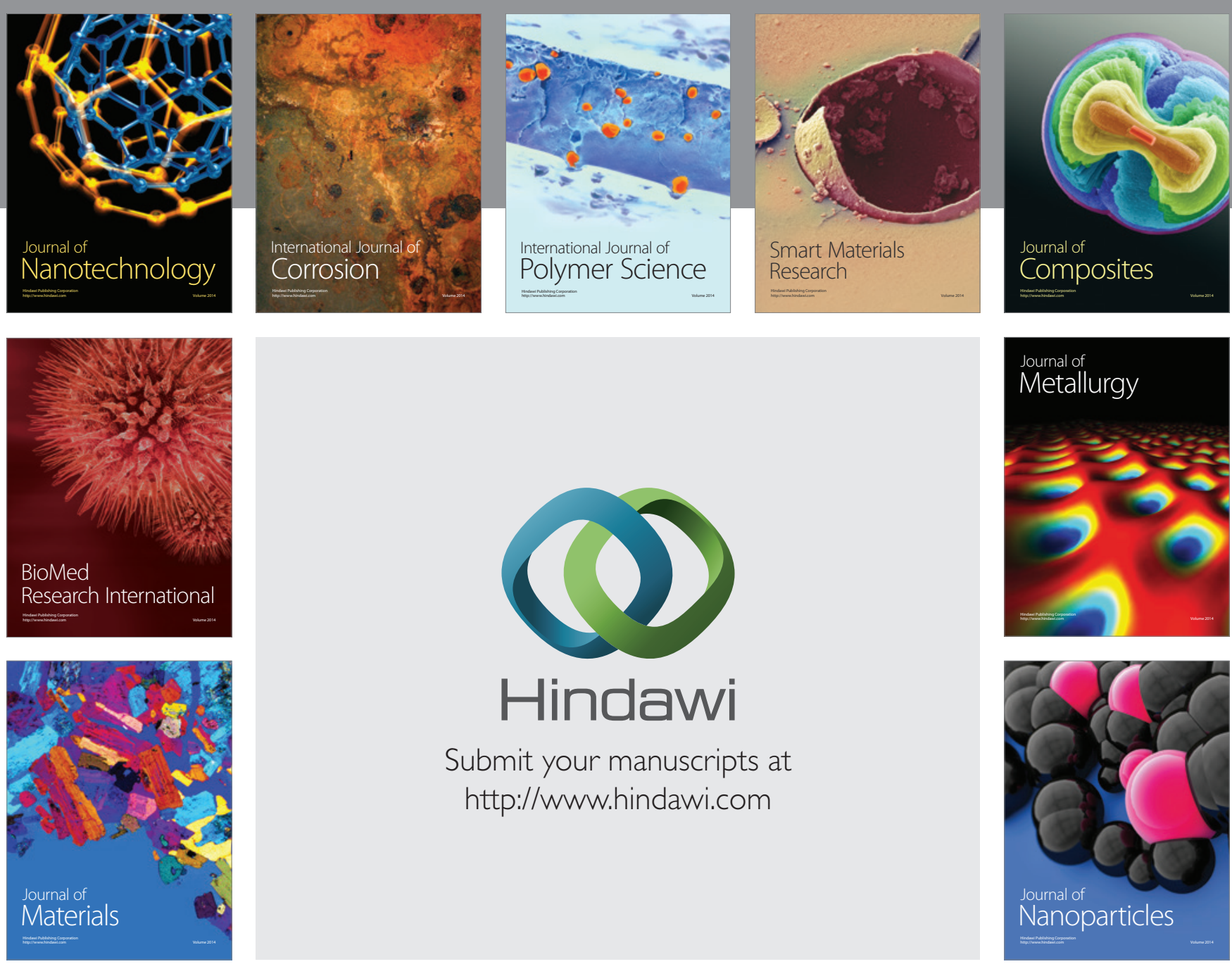

Submit your manuscripts at http://www.hindawi.com
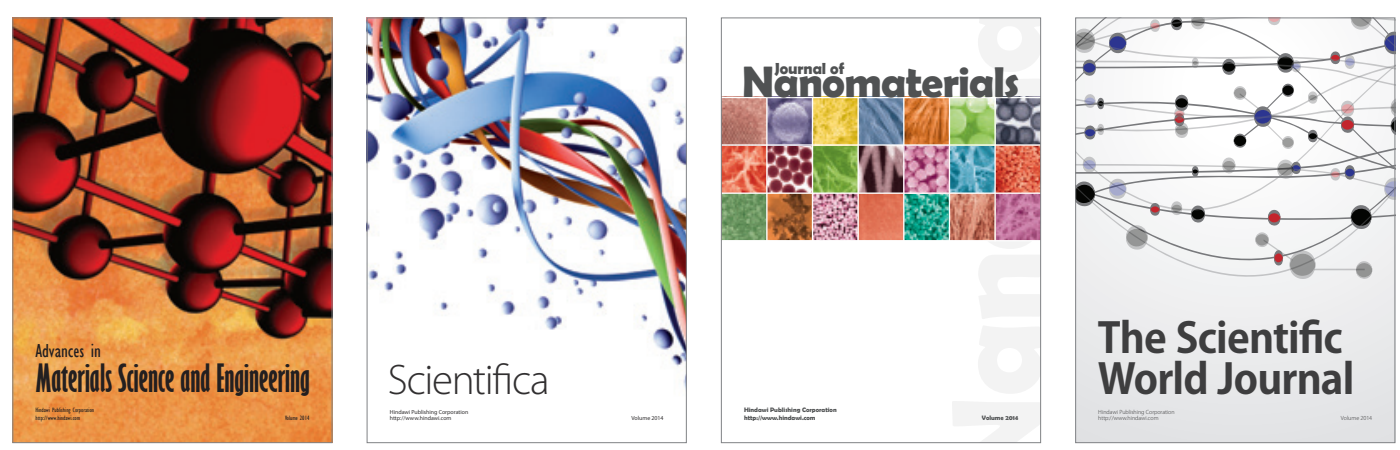

\section{The Scientific World Journal}
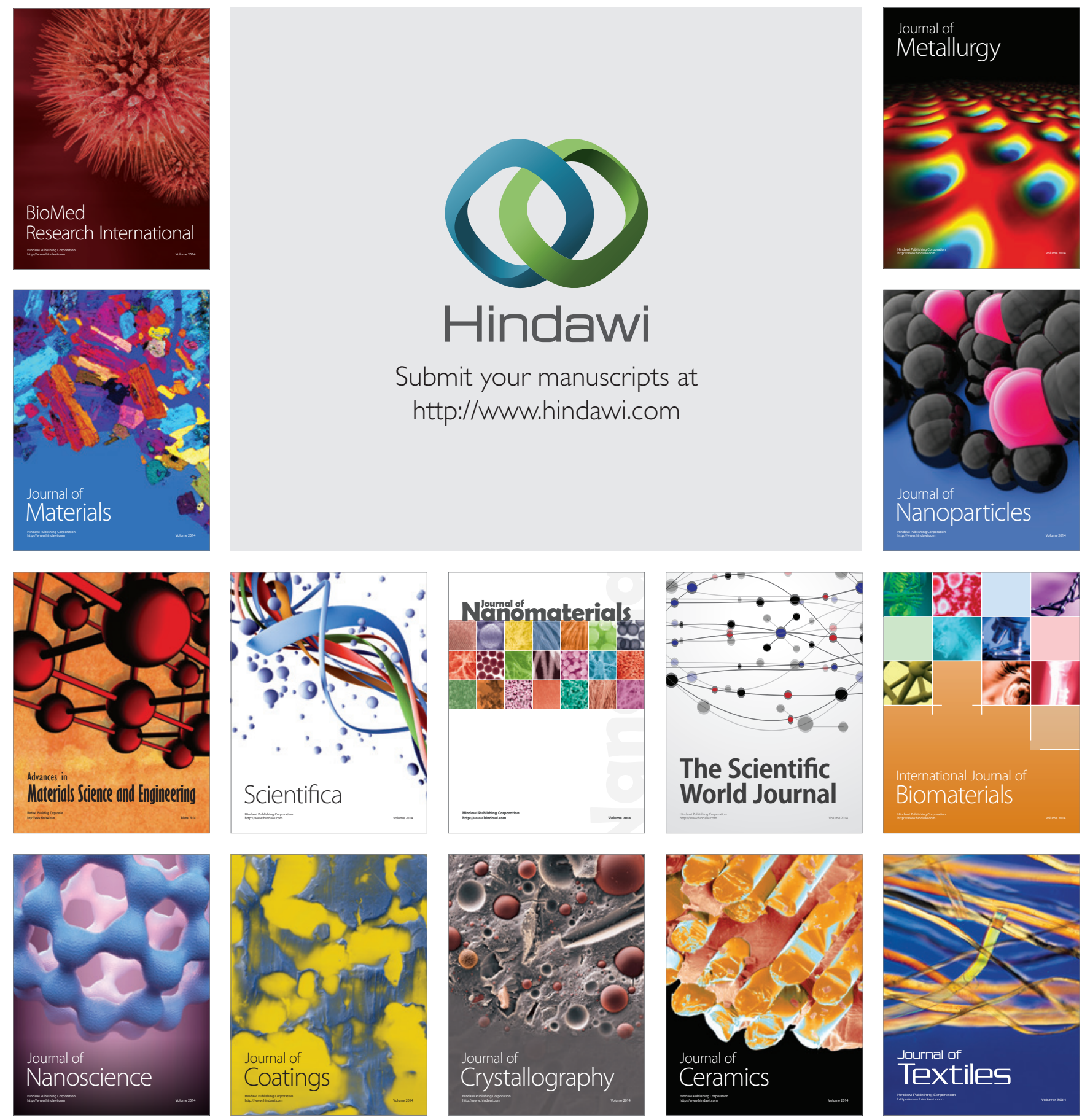